\title{
A monitorização da hipóxia fetal nas gestações complicadas pelo diabete
}

\author{
Fetal hypoxia and its monitoring in diabetic pregnancies \\ Marilza Vieira Cunha Rudge ${ }^{1}$, Iracema de Mattos Paranhos Calderon ${ }^{2}$
}

Introdução

No passado, antes da implantação universal de programas perinatais para o diagnóstico e conduta do diabete na gravidez, a natimortalidade e a mortalidade perinatal (MPN) eram complicações comuns nessas gestações. O entendimento da importância do rígido controle glicêmico materno e do cuidado da mãe e do recém-nascido melhorou o resultado perinatal ${ }^{1}$. Em 1990, a declaração de St. Vincent, publicada pela OMS e pela Federação Internacional de Diabete ${ }^{2}$, instituiu, como meta a ser cumprida em cinco anos, que o resultado da gestação diabética deveria se aproximar da população de grávidas normais. Apesar dos avanços no resultado perinatal em diabéticas é questionável se esta meta foi atingida. A MPN em diabéticas clínicas é ainda cerca de dez vezes maior que a observada em gestantes normais, indicando que a meta da declaração de St. Vincent está longe de ser atingida.

A evolução na conduta obstétrica de resolver no termo as gestações complicadas pelo diabete diminuiu a morbidade e a mortalidade decorrente da prematuridade ${ }^{1,3}$. Por outro lado, esse prolongamento da gestação aumentou o risco de macrossomia e de hipóxia intra-uterina. A macrossomia fetal é uma das complicações mais freqüentes em diabéticas e tem correlação com os níveis de insulina e eritropoetina no líquido amniótico, sugerindo que quanto mais o peso fetal se afasta da normalidade, maior é o risco de hipóxia crônica ${ }^{4}$. Estudos clínicos e experimentais mostraram que tanto a hiperglicemia como a hiperinsulinemia fetais podem, de modo independente, causar hipóxia fetal por aumentar o consumo fetal de oxigênio.

O risco de hipóxia intra-uterina justifica a necessidade de se estabelecer protocolo(s) adequado(s) de avaliação do bem-estar fetal (BEF) nas gestações complicadas pelo diabete. Apesar disso, não há consenso na literatura sobre o melhor teste, a freqüência e a idade gestacional da avaliação e o tipo de diabete - tipo 1, 2 e/ou gestacional (DMG) de maior risco para sofrimento fetal. A maioria dos estudos sugere que vários aspectos da avaliação fetal em diabéticas são prejudicados pelo atraso na maturação do $\mathrm{SNC}^{5}$.

A avaliação intra-uterina do filho de mãe diabética é recomendada pelo risco elevado de MPN. O objetivo da avaliação do BEF é detectar alterações de parâmetros biofísicos, que estão associados com o comprometimento fetal e que necessitam de intervenção. Entretanto, não há ensaios clínicos controlados que comprovem a validade da avaliação antenatal na melhora do resultado perinatal e, também, não está definido qual o melhor método de avaliação do BEF nessas gestações de risco.

Na verdade, o risco de MPN no DMG é baixo e estudos para demonstrar o efeito de estratégias para modificá-lo necessitariam de número elevado de gestantes. Por exemplo, se a MPN é de 10/1000 nascimentos, uma intervenção para demonstrar 50\% de redução no risco, ou seja, atingir índices de 5/1000, com poder estatístico de $80 \%$ na detecção de uma diferença significativa, com erro alfa de 0,05, exige a randomização de 10.400 gestantes (5.200 em cada grupo). Por isso, não é surpresa a inexistência de evidências científicas para as estratégias de avaliação antenatal em portadoras de $\mathrm{DMG}^{6}$.

\section{Monitorização do BEF em gestantes diabéticas}

A contagem materna dos movimentos fetais $(\mathrm{MF})$ é o mais simples e mais antigo método, porém, tem considerável percentagem de erro. A proporção de falso-negativos é baixa, menos de $1 \%$, mas os falsos positivos estão ao redor de $60 \%$. Quando observados pela ultra-sonografia, apenas um terço dos MF são percebidos pela mãe. Apesar da dificuldade em se estabelecer o padrão de norma-

1 Professora Titular de Obstetrícia - Faculdade de Medicina de Botucatu - Universidade Estadual Paulista “Julio Mesquita Filho" - UNESP - Botucatu (SP) - Brasil. Pró-reitora de Pós-Graduação da UNESP.

2 Professora Livre-docente de Obstetrícia - Faculdade de Medicina de Botucatu - Universidade Estadual Paulista “Julio Mesquita Filho" - UNESP Botucatu (SP) - Brasil.

Correspondência: Marilza Vieira Cunha Rudge

Rua General Telles, 1396, Apto. 81 - Centro - Botucatu - SP - 18602-120 - Fone: (14) 3882-0898 - FAX: (14) 3882-2004 - e-mail: mrudge@fmb.unesp.br / propg@reitoria.unesp.br 
lidade nos filhos de mães diabéticas, a redução dos MF pode significar hipóxia e preceder o óbito intra-uterino ${ }^{7}$. Em nosso serviço temos usado a contagem do número de MF, em três períodos diários, como método de rastreamento do $\mathrm{BEF}$ em diabéticas.

A cardiotocografia (CTG) anteparto, método não invasivo, simples, rápido, de fácil interpretação e de baixo custo, não tem unanimidade em relação a critérios de uso e valor preditivo em gestantes diabéticas ${ }^{5}$. O risco potencial de hipoglicemia, cetoacidose e/ou outros distúrbios metabólicos limita o valor prognóstico do teste, pela dificuldade na previsão do tempo em que o feto pode ser considerado saudável após um resultado satisfatório. O teste negativo tem poder prognóstico alto, porém o retardo na maturação do SNC pode interferir na idade gestacional de definição das atividades biofísicas fetais, diferenciar o padrão de normalidade de fetos normais e dificultar a interpretação da $\mathrm{CTG}^{5}$. Alguns estudos têm evidenciado que mesmo um traçado normal na CTG pode se associar a índices baixos de Apgar em 20\% dos $\operatorname{casos}^{8}$ e óbito neonatal ${ }^{5}$. Em contrapartida, resultados anormais na CTG relacionam-se a controle glicêmico materno inadequado e são mais freqüentes em recém-nascidos grandes para a idade gestacional ${ }^{9}$.

A CTG computadorizada é outra técnica usada na avaliação do BEF em gestantes diabéticas. Aqui, também, os traçados demonstram atraso na maturação biofísica fetal, caracterizado por ausência de episódios de alta variação e por diferenças nas variações de curto prazo, na FCF basal, na aceleração e na freqüência dos $\mathrm{MF}^{10}$. Nomura et al. ${ }^{11}$, neste fascículo, observaram menor tempo médio nos episódios de alta variação nos fetos grandes para a idade gestacional. Esses resultados não só ratificam o atraso na maturação do SNC, como também indicam a necessidade de modificar alguns critérios para adequar a interpretação da CTG computadorizada nas gestações complicadas pelo diabete ${ }^{10,12}$.

Manning idealizou o perfil biofísico fetal para aumentar o valor preditivo da CTG anteparto. Este método avalia os MF, os movimentos respiratórios (MR), o tônus e reatividade cardiaca fetal, mas pouco se sabe sobre sua aplicabilidade em gestantes diabéticas. Os MR são observados em idade gestacional mais precoce e acontecem em maior número, diretamente relacionados ao volume do líquido amniótico. O ciclo da movimentação fetal é interrompido e parece se relacionar aos níveis glicêmicos maternos ${ }^{4}$.

As análises dos efeitos do diabete nas características biofísicas fetais são conflitantes e dificultam a definição de parâmetros biofísicos de normalidade nas diferentes idades gestacionais. Admite-se que a hiperglicemia está associada a atraso na maturação do SNC, coordenador das atividades biofísicas do feto, e a estímulo da atividade respiratória, com aumento no número de MR. Entretanto, não está estabelecido como essas alterações interferem na interpretação de testes biofísicos do BEF. O controle metabólico estrito e a vigilância fetal precoce e constante associam-se ao melhor prognóstico perinatal, mas o nivel ideal de glicemia e o melhor teste anteparto ainda são duas áreas de indefinição e, sem dúvida, frentes promissoras e necessárias de pesquisa.

Considerando que o mecanismo patogênico da hipóxia fetal no diabete é mal entendido, o modelo hipotético de adaptação placentária em resposta à hipóxia ${ }^{13}$ parece promissor. De acordo com seus proponentes, a hiperglicemia leva à hipóxia intra-uterina e estimula a angiogênese placentária, com conseqüente aumento na superficie de troca materno-fetal, para garantir aporte adequado de oxigênio ao feto. Associam-se a este conceito estudos específicos de morfometria, evidenciando que esta vicariância placentária é diretamente dependente dos níveis glicêmicos maternos e intra-uterinos*. Neste contexto, a evolução dos índices de pulsatilidade das artérias umbilicais, avaliada pela dopplervelocimetria, poderia refletir as condições de glicemia e oxigenação placentárias e intra-uterinas e auxiliar na avaliação do BEF nestas gestações**.

\section{Considerações finais}

A escassez de dados sobre os testes de avaliação do BEF em gestações complicadas por diabete resulta na proposição de diversos tipos de protocolos, que variam de acordo com o tipo de diabete, o tratamento instituído e a idade gestacional. Tais protocolos propõem tanto a não-necessidade de avaliação do BEF nas portadoras de DMG controlado com dieta, até avaliações mais freqüentes nas diabéticas clínicas de classes mais avançadas (classes FRH) - duas vezes por semana, desde a $28^{a}$ semana de gestação. O $4^{\circ}$ Workshop Internacional de DMG (1997) recomenda que o início e a freqüência da avaliação fetal sejam determinados pela gravidade da hiperglicemia e do quadro clínico materno. Assim, não é surpresa a divergência entre os vários protocolos da literatura, instituídos de acordo com as características das gestantes e do próprio serviço. 
O protocolo adotado no Setor de Investigação do Diabete Perinatal, do Departamento de Ginecologia e Obstetrícia da Faculdade de Medicina de Botucatu-UNESP, está descrito abaixo: para avaliação do BEF são utilizados, além da contagem do número de MF (CMF), a CTG basal, complementada, quando necessário, pelo Estímulo Vibro-Acústico (EVA) (CTG/EVA), o perfil biológico fetal e a dopplervelocimetria das circulações uterina, placentária e cerebral fetal. Todas gestantes diabéticas são orientadas para realizarem a CMF, em três períodos diários, a partir da $28^{\text {a }}$ semana de gestação. As diabéticas com controle glicêmico adequado $(\mathrm{MG}<120 \mathrm{mg} / \mathrm{dL})$ são submetidas a avaliação semanal do BEF, iniciada por CMF e CTG/Estímulo Vibro-Acústico (EVA), após a $28^{\mathrm{a}}$ semana de gestação. Aquelas com controle glicêmico inadequado ( $\mathrm{MG} \geq 120 \mathrm{mg} / \mathrm{dL})$, comportamento não físiológico da necessidade de insulina ou portadoras de vasculopatia são submetidas a avaliações mais freqüentes do BEF - no mínimo, CMF CTG/EVA, duas vezes por semana, a partir da $28^{a}$ semana de gestação. O PBF é realizado sempre que a CTG/EVA tem resultado suspeito ou alterado e a dopplervelocimetria das circulações placentária e cerebral fetal é repetida com intervalo máximo de 15 dias, podendo variar de acordo com o quadro clínico e a gravidade do diabete materno.

Estas recomendações não foram testadas em ensaios clínicos randomizados e, portanto, não têm evidência científica de risco ou beneficio materno-fetal. Até que isso ocorra, seria prudente o uso de todas as técnicas disponíveis, na tentativa de evitar possiveis falhas diagnósticas na avaliação do BEF e prevenir desfechos indesejáveis nestas gestações de risco.

\section{Referências}

1. Rudge MV, Calderon IM, Ramos MD, Abbade JF, Rugolo LM. Perinatal outcome of pregnancies complicated by diabetes and by maternal daily hyperglycemia not related to diabetes. A retrospective 10-year analysis. Gynecol Obstet Invest. 2000;50(2):108-12.

2. World Health Organization. International Diabetes Federation. Diabetes care and research in Europe: the Saint Vincent declaration. Diabet Med. 1990;7(4):360.

3. Yamamoto RM, Francisco RPV, Miyadahira S, Banduki Neto JD, Zugaib M. Avaliação da vitalidade fetal em gestantes diabéticas: análise dos resultados neonatais. Rev Bras Ginecol Obstet. 2000;22(9):557-66.

4. Teramo KA, Hiilesmaa VK. Fetal hypoxia and its monitoring in pregestational diabetic pregnancies. In: Djelmis J, Desoye G, Ivanisevic M, editors. Diabetology of pregnancy. Basel: Karger; 2005. p. 222-9.

5. Cabero-Roura L, Cerqueira, MJ. Care of the pregnant diabetic woman. In: van Assche FA, editor. Diabetes and pregnancy. London: Elsevier; 2004. p. 85-95.

6. Rosenn BM, Miodovnik M. Fetal biophysical and biochemical testing. In: Reece EA, Coustan DR, Gable SG, editors. Diabetes in women. $3^{\text {rd }}$ ed. Philadelphia: Lippincott Willians \& Wilkins; 2004. p. 335-44.

7. Pereira BG, Faundes A, Parpinelli MA, Gama JCG. Percepção materna de movimentos fetais como método de avaliação da vitalidade fetal em gestantes diabéticas. Rev Bras Ginecol Obstet. 1999;21(10):579-84.

8. Rudge MVC, Calderon IMP, Ramos MD, Suetake H, Sá ACJ, Cruz MA. Avaliação da vitalidade dos fetos de gestantes diabéticas: análise cardiotocográfica. Rev Bras Ginecol Obstet. 1994;5(4):201-7.

9. Mascaro MS, Calderon IMP, Costa RAA, Maestá I, Bossolan G, Rudge MVC. Cardiotocografia anteparto e prognóstico perinatal em gestações complicadas pelo diabete: influência do controle metabólico materno. Rev Bras Ginecol Obstet. 2002;24(9):593-9.

10.Tincello D, White S, Walkinshaw S. Computerised analysis of fetal heart rate recordings in maternal type I diabetes mellitus. BJOG. 2001;108(8):853-7.

11.Nomura RMY, Costa VN, Sakamoto K, Maganha CA, Miyadahira S, Zugaib M. Cardiotocografia computadorizada em gestações complicadas pelo diabete melito pré-gestacional: padrões de freqüência cardiaca em fetos grandes para idade gestacional. Rev Bras Ginecol Obstet. 2005;27(12):712-8.

12.Ruozi-Berretta A, Piazze JJ, Cosmi E, Cerekja A, Kashami A, Anceschi MM. Computerized cardiotocography parameters in pregnant women affected by pregestational diabetes mellitus. J Perinat Med. 2004;32(5):426-9.

13.Desoye G, Myatt L. The placenta. In: Reece EA, Coustan DR, Gabbe SG, editors. Diabetes in women. Adolescence, pregnancy and menopause. Philadelphia: Lippincott Williams \& Wilkins; 2004. p. 147-57.

* Calderon IMP, Amorin RL, Damasceno DC, Costa RAA, Brasil MAM, Rudge MVC. Morphometric study of placental villi and vessels in women with diurnal hyperglycemia and gestational and clinical diabetes. Placenta. [submetido para publicação].

** Calderon IMP, Consonni M, Magalhães CG, Amorin RL, Brasil MAM, Rudge MVC. Doppler velocimetric study of umbilical artery and morphometric changes of placental villi and vessels in pregnant women with hyperglycemia. BJOG. [submetido para publicação]. 